\title{
Correlações entre a densitometria do cristalino através da imagem de Scheimplufg, tempo e gasto de energia na cirurgia de facoemulsificação
}

\author{
Correlations between densitometry of the lens by Scheimplufg \\ imaging, time factor and energy (power US) in phacoemulsification
}

Bruno de Freitas Valbon', Ana Laura Canedo², Renata Siqueira Silva ${ }^{3}$, Leonardo Pimentel ${ }^{4}$, Guillermo Velaverde ${ }^{5}$, Renato Ambrósio Junior ${ }^{6}$

\section{Resumo}

Objetivo: Correlacionar a densitometria do cristalino (PNS - patient nuclear score) por meio da imagem de Scheimplufg com o tempo de facoemulsificação e o gasto de energia. Métodos: Estudo observacional prospectivo, envolvendo 22 olhos de 22 pacientes que se submeteram à cirurgia de catarata por facoemulsificação com implante de lente intraocular. A densitometria do cristalino (PNS) foi medida através da tomografia de segmento anterior e córnea (Pentacam) no pré-operatório com a imagem de Scheimplufg. O tempo de facoemulsificação e o gasto de energia foram verificados e anotados ao término de cada cirurgia. $\mathrm{O}$ teste de correlação de Pearson foi utilizado para análise estatística através do programa Bioestat 5.0. Foi considerado como estatisticamente significante $\mathrm{p}<0,05 \%$. Resultados: As correlações entre PNS x Tempo de Facoemulsificação (s) e PNS x Gasto de Energia obtiveram respectivamente um p $<0,0050$ e RP (Coeficiente de Pearson) de 0,5757 e p $<0,0029$ e RP (Coeficiente de Pearson) de 0,6034. Conclusão: Observamos uma forte associação entre o método objetivo de classificação da catarata através da imagem de Scheimplufg (PNS), tempo e gasto de energia. Assim há possibilidade de realizarmos um planejamento cirúrgico pré-operatório baseado em métodos objetivos da classificação da catarata, reduzindo a fluídica, energia e tempo na cirurgia de facoemulsificação.

Descritores: Densitometria; Facoemulsificação/métodos; Diagnóstico por imagem/métodos; Fator tempo

\section{Abstract}

Purpose: To evaluate and to correlate the PNS (patient nuclear score) and time and energy (power US) in patients prior to and following phacoemulsification with intraocular lens implantation. Methods: This is a prospective study. Twenty two consecutive patients (22 eyes) that underwent phacoemulsification surgery and intraocular lens implantation were enrolled. Full ophthalmologic clinical assessment including Pentacam (Oculus, Pentacam) measurements were performed in all cases prior to surgery (Pre). Excluison criteria were corneal disease or scars. Results: There are statistically significant difference between PNS and Time with $p<0,0050$ and PNS $x$ Energy with $p<0,0029$. Conclusion: Preoperatively adjusting phaco parameters based on cataract grade helped improve overall efficiency by reducing the amount of energy and fluid used in the eye and reducing overall phaco time.

Keywords: Densitometry; Phacoemulsification/methods; Diagnostic imaging/methods; Time factor

\footnotetext{
1Pós-graduando (Mestrado) da Universidade Federal Fluminense - UFF - Rio de Janeiro (RJ), Brasil;

${ }^{2}$ Fellow de Córnea e Cirurgia Refrativa do Instituto de Olhos Renato Ambrósio - Rio de Janeiro (RJ), Brasil;

${ }^{3}$ Fellowship em Glaucoma no Wills Eye Hospital - Filadélfia - EUA;

${ }^{4}$ Fellow de Cirurgia Refrativa no Instituto de Olhos Renato Ambrósio - Rio de Janeiro (RJ), Brasil;

${ }^{5}$ Professor Adjunto do Departamento de Estatística da UFF - Rio de Janeiro(RJ), Brasil;

${ }^{6}$ Coordenador do Grupo de Estudos de Tomografia e Biomecânica de Córnea do Rio de Janeiro - Rio de Janeiro (RJ), Brasil.

Os autores declaram inexistir conflitos de interesse.
}

Recebido para publicação em: 1/8/2010 - Aceito para publicação em 25/9/2010 


\section{INTRODUÇÃO}

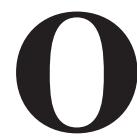

Sistema LOCS III (Lens Opacification Classification System III)(1) é o método mais utilizado para classificação da catarata. Porém, é um método subjetivo, podendo levar a interpretações diferentes, principalmente em cataratas em estágios iniciais. Ainda não existe um método objetivo e com boa reprodutibilidade para graduação da catarata. Além disso, não existe uma forma objetiva de sabermos o quanto de energia necessária será preciso para remoção da mesma na cirurgia de facoemulsificação. O PNS (patient nuclear score) pode ser este método objetivo. E será possível programarmos com eficácia o quanto de energia será necessária durante a cirurgia de facoemulsificação.

O uso de energia (US - ultrasound power) é um importante parâmetro na cirurgia de facoemulsificação. Níveis baixos de US Power podem limitar o perigo às células endoteliais, portanto faz sentido planejar a cirurgia de facoemulsificação com baixos níveis de US de acordo com a densidade da catarata ${ }^{(2,3)}$.

Durante a cirurgia de facoemulsificação é comum cirurgiões alterarem os parâmetros do facoemulsificador de acordo com a densidade da catarata. Pensamos, que o planejamento da cirurgia é mais eficaz e seguro se nos basearmos em métodos objetivos como o PNS.

\section{Métodos}

Foram avaliados 22 olhos de 22 pacientes com diagnóstico de catarata que foram submetidos à cirurgia de facoemulsificação com implante de lente intraocular.

O estudo foi desenvolvido respeitando as normas e orientações estabelecidas na Declaração de Helsinki e as orientações e normas do Comitê de Ética em Pesquisa da Faculdade de Medicina da Universidade Federal Fluminense o qual aprovou sua execução.

Foram critérios de exclusão do estudo a presença de quaisquer doenças do segmento anterior.

Todas as cirurgias foram realizadas pelo mesmo cirurgião, utilizando a incisão córnea clara $(2.7 \mathrm{~mm})$, agente viscoelástico (metilcelulose a $2 \%$ e $4 \%$ ), técnica Stop \& Chop e implante de lente intraocular (LIO) dobrável.

Após o exame oftalmológico de rotina, todos os pacientes inclusos no estudo foram avaliados em midríase pela Tomografia de Segmento Anterior e Córnea (Pentacam, Oculus) que nos fornece o PNS (patient nuclear score) através da imagem de Scheimplufg, classificando a densitometria do cristalino de 1-5.

A figura 1 mostra a classificação do PNS através da imagem de Scheimplufg.
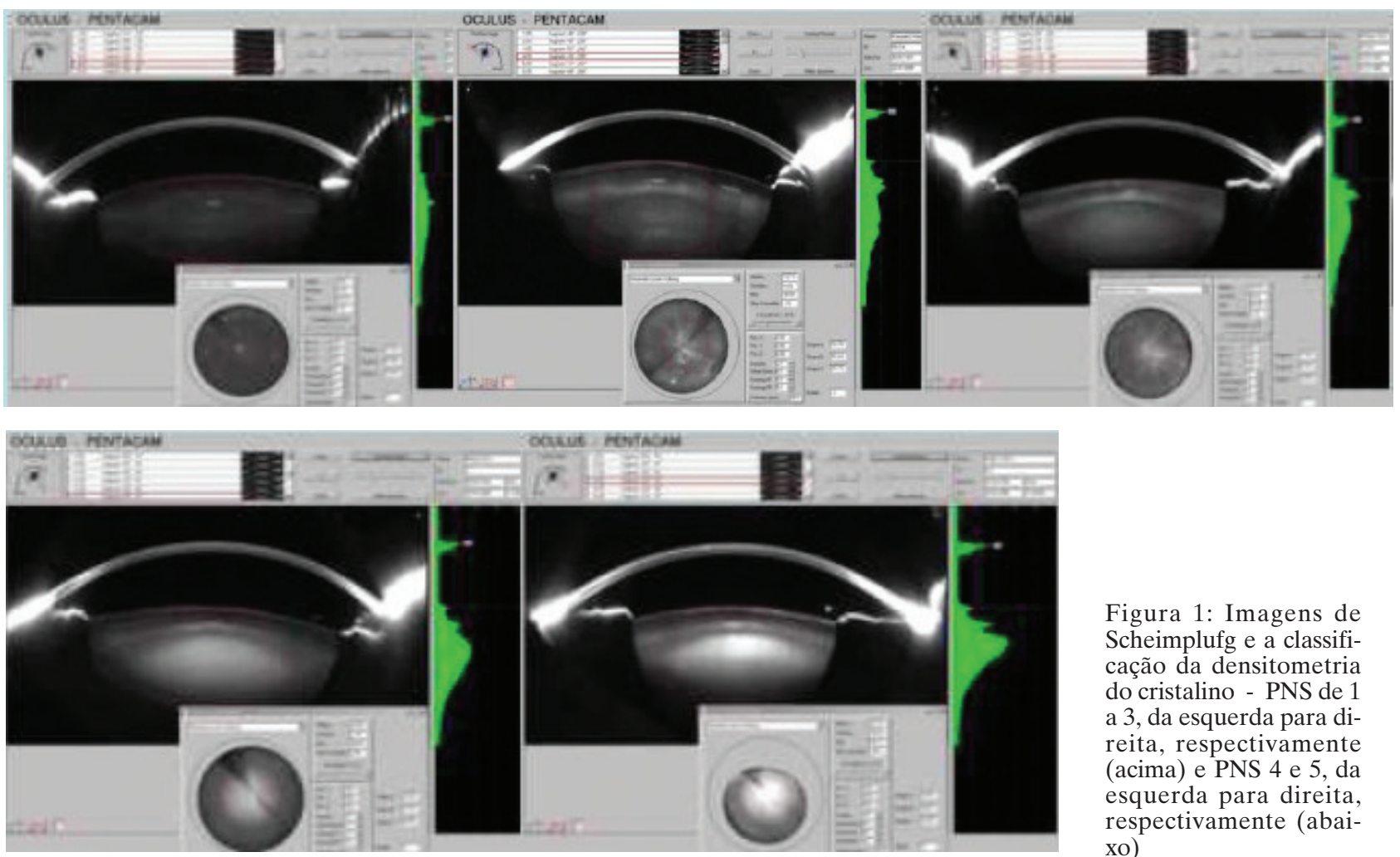

Figura 1: Imagens de Scheimplufg e a classificação da densitometria do cristalino - PNS de 1 a 3, da esquerda para direita, respectivamente (acima) e PNS 4 e 5, da esquerda para direita, respectivamente (abaixo) 


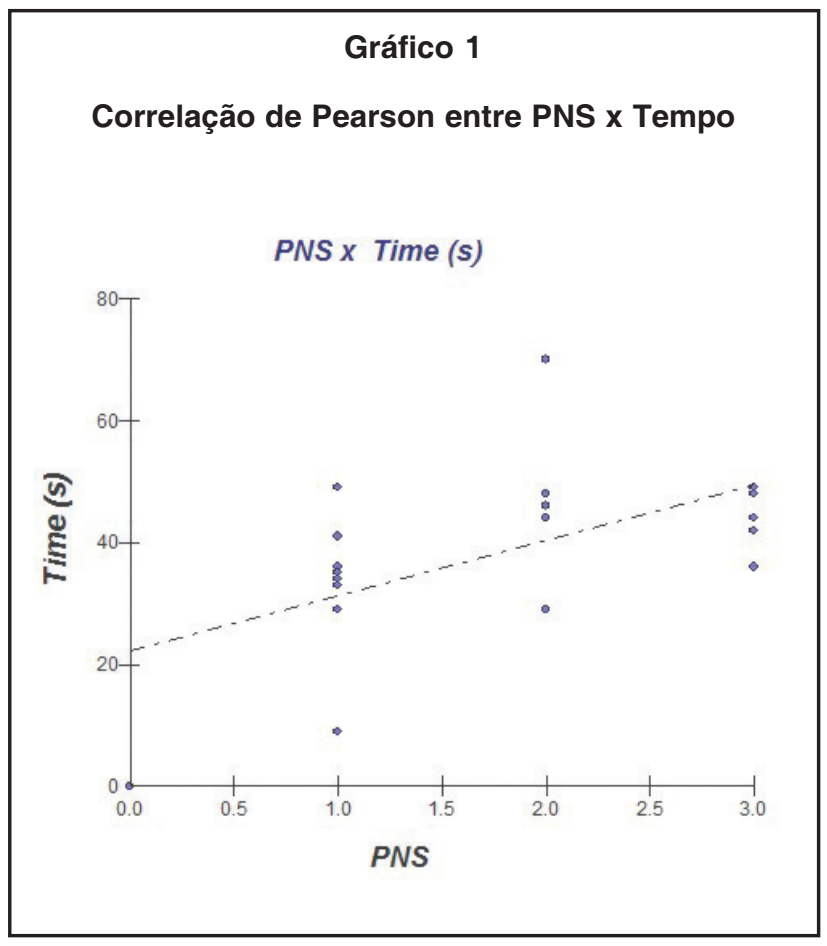

A cirurgia de facoemulsificação foi realizada no aparelho de facoemulsificador Millenium (Bausch \& Lomb).

Ao término de cada cirurgia, o cirurgião responsável verificou e anotou o tempo em segundos e o gasto de energia da cirurgia de facoemulsificação.

OTeste de Kolmogorov-Smirnov foi utilizado para avaliar a distribuição normal. Os dados foram considerados normais, sendo assim a Correlação de Pearson foi a escolhida, e a utilizamos para a correlação das variáveis PNS, tempo e gasto de energia. Foi considerado como estatisticamente significante $\mathrm{p}<0,05 \%$.

\section{Resultados}

As correlações entre as variáveis PNS x Tempo (s) e PNS x Gasto de Energia foram estatisticamente significante, apresentando um $\mathrm{p}<0,0050$ e $\mathrm{p}<0,0029$, respectivamente.

O coeficiente de Pearson foi de 0,5757 para PNS $\mathrm{x}$ tempo(s) e de 0,6034 para PNS x gasto de energia.

Podemos observar no Gráfico 1 a associação entre PNS x tempo e no Gráfico 2 a relação entre PNS x energia, respectivamente.

Há uma forte relação entre as variáveis estudadas. Isso mostra que pode ser um método seguro no planejamento cirúrgico da facoemulsificação.

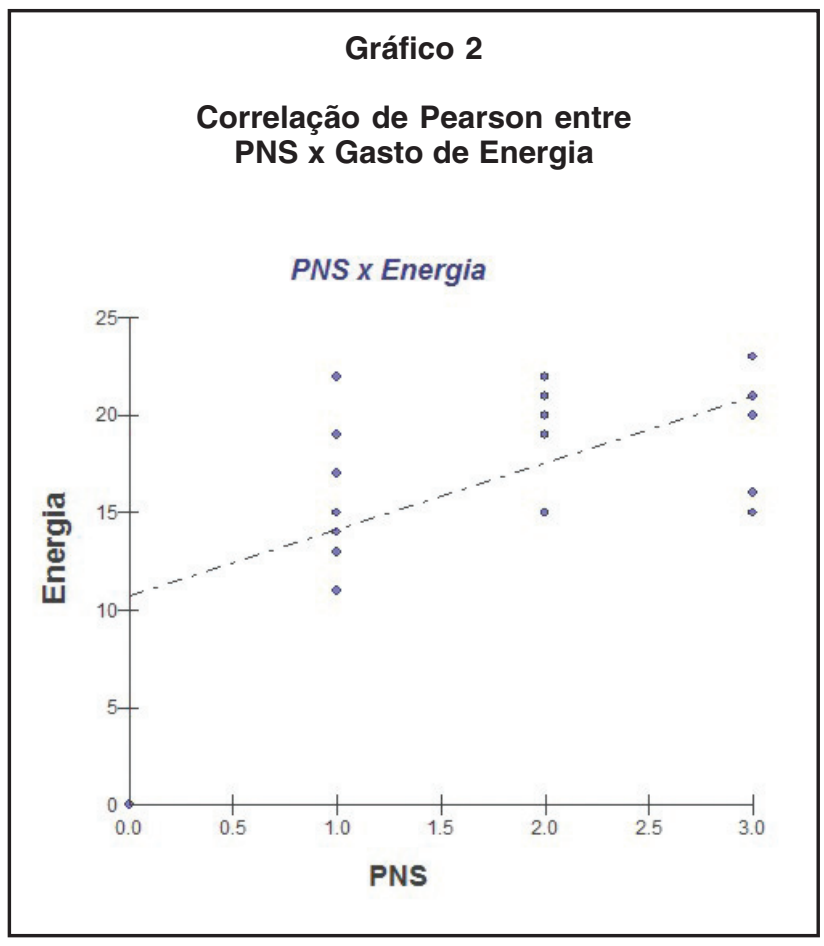

\section{DiscussÃo}

A avaliação objetiva e com boa reprodutibilidade das características pré-operatórias da densidade da catarata nos ajuda a realizar um planejamento cirúrgico eficaz. O sistema mais amplamente utilizado para avaliação da catarata é o LOCS III, porém pode ser interpretado diferentemente por examinadores, sendo um método subjetivo.

Neste estudo, avaliamos a densidade do cristalino através da imagem de Scheimplufg e correlacionamos com o gasto de energia e tempo de facoemulsificação, havendo forte relação entre essas variáveis.

Em geral, os médicos cirurgiões programam a cirurgia de acordo com a densidade do cristalino pelo método subjetivo, já com este novo método objetivo é possível programar com mais eficácia a cirurgia de facoemulsificação, que traz benefícios como diminuição do US (ultrasound Power), havendo menos consumo de energia e consequentemente menos dano às células endoteliais. Além disso, será possível num futuro próximo que os facoemulsificadores tenham em seus programas o planejamento cirúrgico de acordo com a análise objetiva da catarata.

Em recente artigo publicado, Nixon ${ }^{(4)}$ mostrou que há associação entre gasto de energia, uso de BSS e tempo de facoemulsificação entre vários grupos de den- 
sidade de catarata avaliados pela imagem de Scheimplufg, corroborando com nossos resultados.

Nosso estudo é um dos pioneiros que correlaciona a densitometria do cristalino e as variáveis tempo de facoemulsificação e gasto de energia.

Concluímos que existe uma forte associação entre o método objetivo de classificação da catarata através da imagem de Scheimplufg (PNS), tempo e gasto de energia. Assim há possibilidade de realizarmos um planejamento cirúrgico pré-operatório baseado em métodos objetivos da classificação da catarata, reduzindo a fluídica, o gasto de energia e tempo na cirurgia de facoemulsificação.

\section{REFERÊNCIAS}

1. Chylack LT Jr, Wolfe JK, Singer DM, Leske MC, Bullimore MA, Bailey IL, et al. The Lens Opacities Classification System III. The Longitudinal Study of Cataract Study Group. Arch Ophthalmol. 1993;111(6):831-6.

2. Antao SF, Kasaby H. Increased cutting efficiency in phacoemulsification with Sovereign WhiteStar ICE technology. J Cataract Refract Surg. 2008;34(1):173-4.

3. Baradaran-Rafii A, Rahmati-Kamel M, Eslani M, Kiavash V, Karimian F. Effect of hydrodynamic parameters on corneal endothelial cell loss after phacoemulsification. J Cataract Refract Surg. 2009;35(4):732-7. Comment in: J Cataract Refract Surg. 2009;35(12):2178; author reply 2178-9.

4. Nixon DR. Preoperative cataract grading by Scheimpflug imaging and effect on operative fluidics and phacoemulsification energy. J Cataract Refract Surg. 2010;36(2):242-6.

\section{Endereço para correspondência \\ Bruno de Freitas Valbon \\ Rua Conde de Bonfim, $n^{\circ} 211$ apto. 712 - Tijuca \\ CEP 20520-050 - Rio de Janeiro (RJ), Brasil \\ Fone: 55 (21) 8103-7117 \\ E-mail: valbonbruno@ig.com.br}

